\title{
Identifying Atomic Reconstruction at Complex Oxide Interfaces Using Quantitative STEM
}

\author{
Jared M. Johnson ${ }^{1}$, Justin Thompson ${ }^{2}$, S. S. A. Seo ${ }^{2}$, and Jinwoo Hwang ${ }^{1}$ \\ 1. Department of Materials Science and Engineering, The Ohio State University, Columbus, OH 43212 \\ 2. Department of Physics and Astronomy, University of Kentucky, Lexington, KY 40506
}

We present a novel electron microscopy characterization of atomic reconstruction at epitaxial complex oxide interfaces. One of the most important aspects of oxide heterostructures is the discontinuity in polarity at the interface, which can lead to many exciting new properties that cannot be observed in bulk materials, such as metal-insulator transitions and superconductivity (e.g. [1]). The conflict of polarity may also result in atomic and electronic reconstructions at the interface, which may directly affect the transport properties of the interface. However, identifying the exact atomic reconstruction at the interface is a challenging task because the reconstruction is usually confined to an interfacial region thinner than 1 unit cell. Cross-sectional TEM or STEM images can reveal some information about the interfacial reconstruction, but it is difficult to obtain the reconstruction pattern along the depth direction (parallel to the beam) from the projected image. Therefore the identification of interfacial reconstruction requires a technique that can acquire atomic scale 3-dimensional (3D) information.

In this work, we show that the exact stoichiometry and atomic reconstruction at the $\mathrm{KTaO}_{3} / \mathrm{GdScO}_{3}$ interface can be revealed using 3D quantitative STEM. Quantitative STEM measures the STEM annular dark-field signals on an absolute scale, which can be directly compared to simulated images. It enables direct interpretation of the STEM images in terms of the number of atoms and atomic species, and therefore provides information on the exact number and chemistry of the atoms in each atomic column [2]. Recently, Hwang and co-workers have advanced this technique to reveal atomic scale 3D information [3]. By including the probe channeling information in the analysis of quantitative STEM images, the $3 \mathrm{D}$ positions of impurity atoms in the atomic columns of $\mathrm{SrTiO}_{3}$ have been identified with depth uncertainty less than 1 unit cell. The analysis of quantitative STEM images depends on the comparison between the experimental and simulated images, so the use of STEM image simulation that fully captures the dynamic electron scattering is critical.

$\mathrm{KTaO}_{3}$ and $\mathrm{GdScO}_{3}$ both have polar surfaces and therefore exhibit conflicting net charges at the interface, which drives the atomic/electronic reconstruction. Among many potential mechanisms of the reconstruction, our preliminary result shows that the reconstruction involves an intermixing region that consists of one $\mathrm{K}_{\mathrm{x}} \mathrm{Gd}_{1-\mathrm{x}} \mathrm{O}$ layer and one $\mathrm{Ta}_{\mathrm{y}} \mathrm{Sc}_{1-\mathrm{y}} \mathrm{O}_{2}$ layer [Fig. 1] [4]. The TaScO layer at the bottom shows ordered, alternating Ta- and Sc- rich columns, while the KGdO layer at the top shows an even distribution of atomic column intensities, which implies a more randomized atomic distribution [Fig. 1(A) and (C)]. We will show that by comparing the quantitative STEM images of the interface to multislice STEM simulations, the exact stoichiometry and the atomic ordering (sequence) along the depth direction at each atomic column can be precisely determined [e.g. Fig. 1(B) and (C)]. The atomic reconstruction may also involve the change in the oxygen positions; therefore identifying the oxygen positions at the interface is also crucial. For this, we use position averaged convergent beam electron diffraction (PACBED) that can determine the oxygen atom displacements in a unit cell with a great precision [5]. By comparing the experimental and simulated PACBED patterns, PACBED can also provide precise TEM sample thicknesses [Fig. 1(D)], which is crucial for the analysis of quantitative 
STEM images. The quantitative analysis requires very thin $(<10 \mathrm{~nm})$ TEM samples with damage-free surfaces, which we achieve by using the chemical-mechanical sample polishing technique [6], which provides far superior results compared to samples prepared by FIB.

\section{References}

[1] A. Ohtomo and H. Y. Hwang, Nature 427 (2004) p. 423.

[2] J. M. LeBeau, S. D. Findlay, L. J. Allen, and S. Stemmer, Phys. Rev. Lett. 100 (2008) p. 206101.

[3] J. Hwang, et al., Phys. Rev. Lett. 111 (2013) p. 266101.

[4] J. Thomson et al., Appl. Phys. Lett. 105 (2014) p. 102901.

[5] J. Hwang, J. Y. Zhang, J. Son, and S. Stemmer, Appl. Phys. Lett. 100 (2012) p. 191909.

[6] P. M. Voyles, J. L. Grazul, and D. A. Muller, Ultramicroscopy 96 (2003) p. 251.

(A)

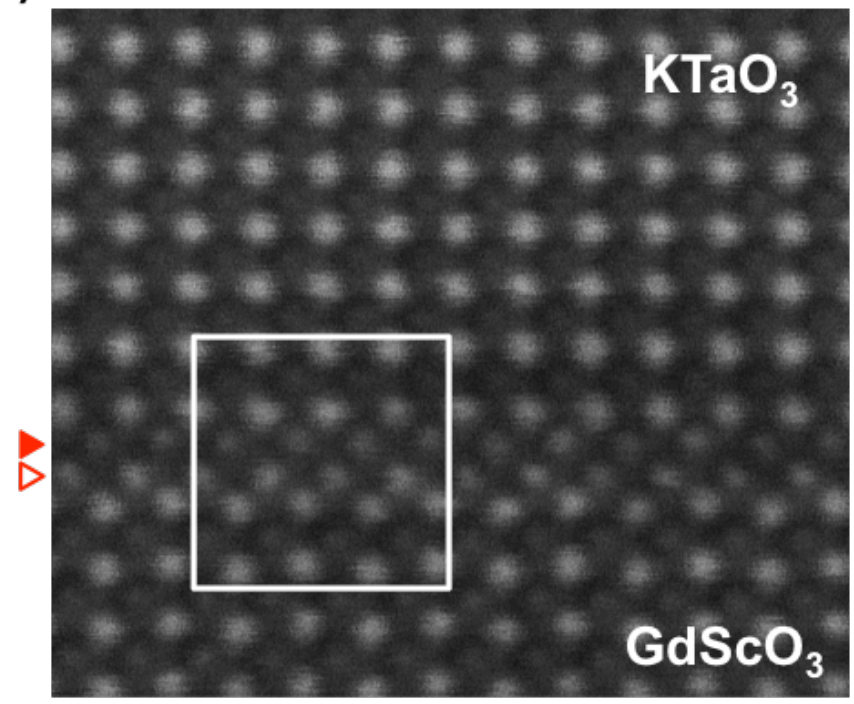

(B)

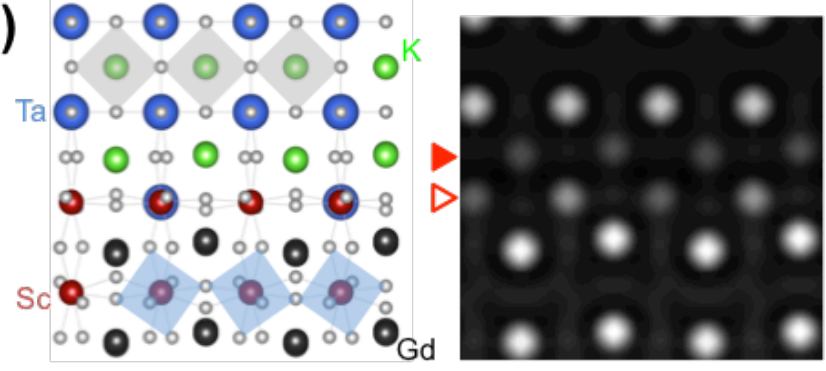

(C)
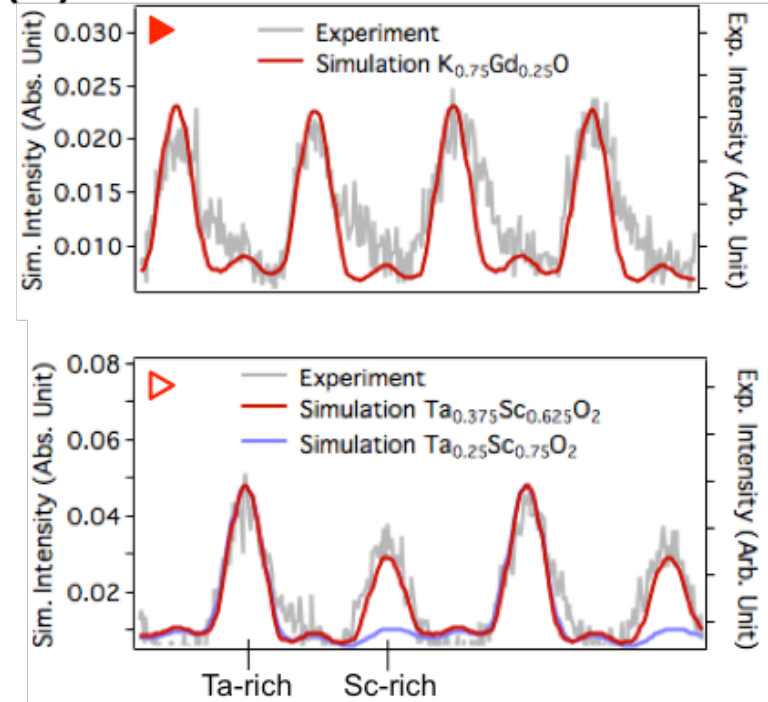

(D)
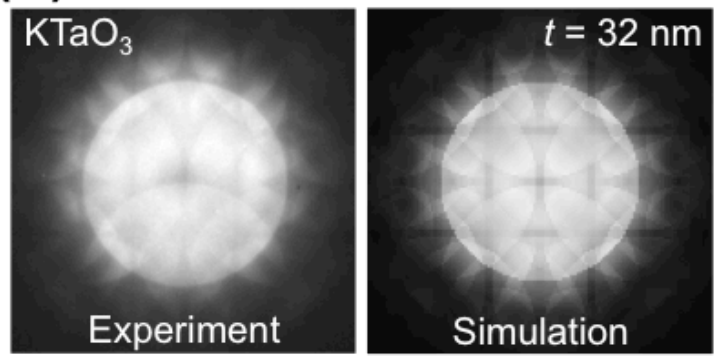

Figure 1. (A) Experimental STEM ADF image of $\mathrm{KTaO}_{3} / \mathrm{GdScO}_{3}$ interface showing atomic reconstruction at the interface [4]. (B) (Left) A model of the squared region in (A), and (right) multislice simulation of the model with $3.2 \mathrm{~nm}$ sample thickness. (C) Qualitative comparison between the experimental and simulated line profiles for (top) $\mathrm{KGdO}$ layer and (bottom) TaScO layer. Fully quantitative comparison will require an experimental image on an absolute scale, and the thickness match between the experimental sample and the model. (D) (Left) Experimental PACBED pattern from the $\mathrm{KTaO}_{3}$ region in (A) that matches (right) the simulated (multislice) PACBED with $32 \mathrm{~nm}$ sample thickness. 